\title{
Activation of Hedgehog pathway promotes fibrogenesis and vascular remodelling in human schistosomiasis
}

Infection with Schistosoma mansoni can cause portal fibrosis and portal hypertension, but the exact mechanisms that underlie these processes are unclear. New research published in Liver International now indicates that schistosome egg antigens (SEAs) stimulate macrophages in the liver to produce Hedgehog $(\mathrm{Hh})$ ligands that, in turn, promote fibrogenesis and vascular remodelling in human schistosomiasis.

"The parasite eggs lodge in tiny blood vessels within the liver. Over time, eggderived factors trigger immune responses that can lead to progressive liver scarring and blood flow abnormalities, which can be life-threatening," explains author Anna Mae Diehl. The researchers investigated whether the Hh pathway regulates this disease course in humans.

Immunohistochemical analysis of liver biopsy samples from 28 patients with varying levels of schistosomiasis

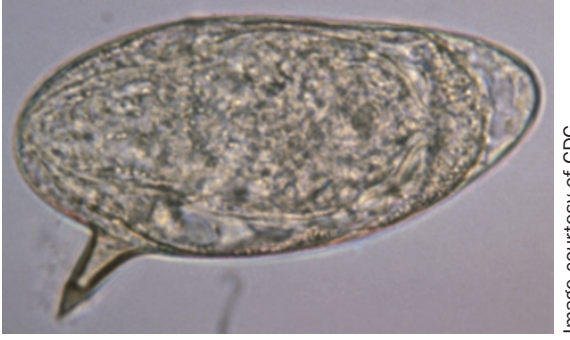

promotion of fibrogenesis and the development of vascular abnormalities; these processes could be blocked in vitro by treatment with $\mathrm{Hh}$ inhibitors.

Looking to the future, the authors are already investigating whether $\mathrm{Hh}$ pathway inhibition can reverse fibrosis and vascular remodelling in experimental models. They say: "If we indeed demonstrate that ... pharmacological inhibitors of Hh pathway could constrain, or even ameliorate, pathological tissue remodelling, this could benefit patients with fibrovascular complications of hepatic schistosomiasis and may be a nonsurgical intervention."

Katrina Ray

Original article Pereira, T. A. Macrophage-derived Hedgehog ligands promotes fibrogenic and angiogenic responses in human schistosomiasis mansoni. Liver Int. doi:10.1111/liv.12016 